\title{
St-closed Submodule
}

\author{
Muna A. Ahmed ${ }^{1}$ and Maysaa R. Abbas ${ }^{2}$ \\ Department of Mathematics, College of Science for Women, Baghdad University, Baghdad-Iraq. \\ ${ }^{1}$ E-mail: math.200600986@yahoo.com. \\ ${ }^{2}$ E-mail: maysaa.alsaher@yahoo.com.
}

\begin{abstract}
Throughout this paper $\mathrm{R}$ represents commutative ring with identity and $\mathrm{M}$ is a unitary left $\mathrm{R}$ module, the purpose of this paper is to study a new concept, (up to our knowledge), named Stclosed submodules. It is stronger than the concept of closed submodules, where a submodule $\mathrm{N}$ of an R-module $\mathrm{M}$ is called St-closed (briefly $\mathrm{N} \leq_{\text {stc }} \mathrm{M}$ ) in $\mathrm{M}$, if it has no proper semi-essential extensions in $\mathrm{M}$, i.e if there exists a submodule $\mathrm{K}$ of $\mathrm{M}$ such that $\mathrm{N}$ is a semi-essential submodule of $\mathrm{K}$ then $\mathrm{N}=\mathrm{K}$. An ideal $\mathrm{I}$ of $\mathrm{R}$ is called St-closed if $\mathrm{I}$ is an St-closed R-submodule. Various properties of St-closed submodules are considered.
\end{abstract}

Keywords: Prime submodules, Essential submodules, Semi-essential submodules, Closed submodules, St-closed submodules, Fully prime modules and fully essential modules.

\section{Introduction}

Let $\mathrm{R}$ be a commutative ring with identity and let $\mathrm{M}$ be a unitary left $\mathrm{R}$-module, and all R-modules under study contains prime submodules. It is well known that a nonzero submodule $\mathrm{N}$ of $\mathrm{M}$ is called essential (briefly $\mathrm{N} \leq \mathrm{e} M)$, if $\mathrm{N} \cap \mathrm{L} \neq(0)$ for each nonzero submodule $\mathrm{L}$ of $\mathrm{M}$ [8], and a nonzero submodule $N$ of $M$ is called semi-essential (briefly $\mathrm{N} \leq_{\text {sem }} \mathrm{M}$ ), if $\mathrm{N} \cap \mathrm{P} \neq(0)$ for each nonzero prime $\mathrm{R}$ - submodule $\mathrm{P}$ of $\mathrm{M}$ [2]. Equivalently, a submodule $\mathrm{N}$ of an R-module $\mathrm{M}$ is called semi-essential if whenever $\mathrm{N} \cap \mathrm{P}$ $=(0)$, then $\mathrm{P}=(0)$ for every prime submodule $\mathrm{P}$ of $\mathrm{M}$ [11], where a submodule $\mathrm{P}$ of $\mathrm{M}$ is called prime, if whenever $r m \in P$ for $r \in R$ and $\mathrm{m} \in \mathrm{M}$, then either $\mathrm{m} \in \mathrm{P}$ or $\mathrm{r} \in\left(\mathrm{P}_{\mathrm{R}}^{:} \mathrm{M}\right)$ [14].

A submodule $\mathrm{N}$ of $\mathrm{M}$ is called closed submodule (briefly $\mathrm{N} \leq_{\mathrm{c}} \mathrm{M}$ ), if $\mathrm{N}$ has no proper essential extensions in $M$, i.e if $N \leq_{e} K$ $\leq \mathrm{M}$ then $\mathrm{N}=\mathrm{K}$ [6]. In our work we introduce a new concept (up to our knowledge), named St-closed submodules, which is stronger than the concept of closed submodules, where a submodule $\mathrm{N}$ of an $\mathrm{R}$-module $\mathrm{M}$ is called St- closed if $\mathrm{N}$ has no proper semi-essential extensions in $M$, i.e if $N \leq$ sem $K \leq M$ then $\mathrm{N}=\mathrm{K}$. This paper consist of three sections, in section one we investigate the main properties of St-closed submodules, such as the transitively property. Also we study the relationships between St-closed submodules, closed submodules and y-closed submodules. In $\mathrm{S}_{2}$ we study the behavior of the class of
St-closed submodules in the class of multiplication modules. In S3 we study modules satisfying the chain conditions on St-closed submodules.

\section{S1: St-closed submodules}

In this section we investigate the main properties of St-closed submodules such as the transitive property. Moreover, we study the relationships between St-closed submodules and other submodules.

\section{Definition (1.1):}

Let $\mathrm{M}$ be an $\mathrm{R}$-module, a submodule $\mathrm{N}$ of $\mathrm{M}$ is called St-closed in $\mathrm{M}$ (briefly $\mathrm{N} \leq_{\text {stc }} \mathrm{M}$ ), if $\mathrm{N}$ has no proper semi-essential extensions in $\mathrm{M}$, i.e if there exists a submodule $\mathrm{K}$ of $\mathrm{M}$ such that $\mathrm{N}$ is a semi-essential submodule of $\mathrm{K}$ then $\mathrm{N}=\mathrm{K}$. An ideal I of $\mathrm{R}$ is called an St-closed, if it is St-closed R-submodule.

\section{Examples and Remarks (1.2):}

1) Consider the $Z$-module $M=Z_{8} \oplus Z_{2}$. In this module there are eleven submodules which are $\langle(\overline{0}, \overline{0})\rangle,\langle(\overline{1}, \overline{0})\rangle,\langle(\overline{0}, \overline{1})\rangle$, $\langle(\overline{1}, \overline{1})\rangle, \quad\langle(\overline{2}, \overline{0})\rangle, \quad\langle(\overline{2}, \overline{1})\rangle, \quad\langle(\overline{4}, \overline{0})\rangle$, $\langle(\overline{4}, \overline{1})\rangle,\langle(\overline{0}, \overline{1}),(\overline{4}, \overline{0})\rangle,\langle(\overline{2}, \overline{0}),(\overline{4}, \overline{1})\rangle$, and $\mathrm{M}$. The submodules $\langle(\overline{0}, \overline{1})\rangle,\langle(\overline{4}, \overline{1})\rangle$, and $\mathbf{M}$ are St-closed in $\mathrm{M}$, since they have no proper semi-essential extensions in $\mathrm{M}$. On the other hand, the submodules $\langle(\overline{0}, \overline{0})\rangle, \quad\langle(\overline{1}, \overline{1})\rangle, \quad\langle(\overline{1}, \overline{0})\rangle, \quad\langle(\overline{2}, \overline{0})\rangle$, $\langle(\overline{2}, \overline{1})\rangle,\langle(\overline{4}, \overline{0})\rangle,\langle(\overline{0}, \overline{1}), \quad(\overline{4}, \overline{0})\rangle$, and $<(\overline{2}, \overline{0}), \quad(\overline{4}, \overline{1})\rangle, \quad$ are not $\quad$ St-closed 
submodules in $\mathrm{M}$, since they have semi-essential extensions in $\mathrm{M}$.

2) Every R-module $M$ is an St-closed submodule in $\mathrm{M}$.

3) (0) may not be St-closed submodule of $M$, for example $(\overline{0})$ is not St-closed submodule in the $\mathrm{Z}$-module, $\mathrm{Z}_{2}$.

4) If a submodule $N$ of an R-module $M$ is a semi-essential and an St-closed, then $\mathrm{N}=\mathrm{M}$.

5) If $\mathrm{N}$ is an St-closed submodule in $\mathrm{M}$ then $\left(\mathrm{N}_{\mathrm{R}}^{\vdots} \mathrm{M}\right)$ need not be St-closed ideal in $\mathrm{R}$, for example; $(\overline{8})$ is an St-closed submodule in the $\mathrm{Z}$-module $\mathrm{Z}_{24}$, while $\left((\overline{8})_{\mathrm{Z}} \mathrm{Z}_{24}\right)=8 \mathrm{Z}$ is not St-closed ideal in Z.

6) A direct summand of an R-module $M$ is not necessary St-closed submodule in $\mathrm{M}$, for example: Consider the $\mathrm{Z}$-module, $\mathrm{Z}_{12}$, where $\quad Z_{12}=(\overline{3}) \oplus(\overline{4})$. The direct summand $(\overline{4})=\{\overline{0}, \overline{4}, \overline{8}\}$ is an St-closed submodule in $\mathrm{Z}_{12}$, since $(\overline{4})$ has no proper semi-essential extensions in $Z_{12}$. But the direct summand $(\overline{3})=\{\overline{0}, \overline{3}, \overline{6}, \overline{9}\}$ of $Z_{12}$ is not St-closed submodule since $(\overline{3})$ is a semi-essential submodule of $Z_{12}$. Also the Z-module, $\mathrm{Z}_{36}=(\overline{4}) \oplus(\overline{9})$, it is clear that $(\overline{9})$ is a direct summand of $Z_{36}$ but not St-closed submodule in $\mathrm{Z}_{36}$.

7) Let $\mathrm{M}$ be an $\mathrm{R}$-module, if $\mathrm{M}=\mathrm{A} \oplus \mathrm{B}$, then even though $\mathrm{A}$ or $\mathrm{B}$ or both of them are prime submodules of $\mathrm{M}$, then neither $\mathrm{A}$ nor $\mathrm{B}$ are necessary St-closed submodules in $\mathrm{M}$. For example: the Z-module $\mathrm{Z}_{30}=(\overline{5}) \oplus(\overline{6})$ $=(\overline{2}) \oplus(\overline{15})$, both of $(\overline{2})$ and $(\overline{5})$ are prime submodules of $Z_{30}$ and direct

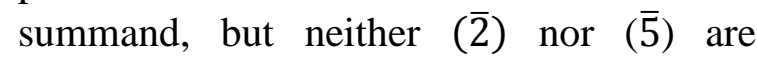
St-closed submodules in $\mathrm{Z}_{30}$. In fact both of $(\overline{2})$ and $(\overline{5})$ are semi-essential submodules of $\mathrm{Z}_{30}$.

8) Let $\mathrm{M}$ be an R-module, and let $\mathrm{A}$ be an St-closed submodule of $M$. If $B$ is $a$ submodule of $\mathrm{M}$ such that $\mathrm{A} \cong \mathrm{B}$, then it is not necessary that $B$ is an St-closed submodule in $M$. For example, the $\mathrm{Z}$-module $\mathrm{Z}$ is an St-closed submodule in $\mathrm{Z}$, and $Z \cong 3 Z$, but $3 Z$ is not St-closed submodule in $Z$, since $3 Z$ is a semiessential submodule of $Z$.

Remarks (1.3):

1) Every St-closed submodule in an $\mathrm{R}$-module $\mathrm{M}$ is a closed submodule in $\mathrm{M}$.
Proof (1):

Let $\mathrm{N}$ be an St-closed submodule in $\mathrm{M}$, and let $\mathrm{K} \leq \mathrm{M}$ with $\mathrm{N} \leq_{\mathrm{e}} \mathrm{K} \leq \mathrm{M}$. Since $\mathrm{N} \leq_{\mathrm{e}} \mathrm{K}$, then $\mathrm{N} \leq_{\text {sem }} \mathrm{K}$ [2, Example (2), P.49]. But $\mathrm{N}$ is an St-closed submodule in $\mathrm{M}$, thus $\mathrm{N}=\mathrm{K}$, that is $\mathrm{N}$ is a closed submodule in $\mathrm{M}$.

The converse is not true in general, for example: In the $\mathrm{Z}$-module $\mathrm{Z}_{24}$ we note that $(\overline{3})$ is a closed submodule in $Z_{24}$, but it is not St-closed. Also $(\overline{9})$ is a closed submodule in $Z_{36}$, but it is not St-closed in $Z_{36}$.

2) Let $N$ be an St-closed submodule of $M$. If $\mathrm{B}$ is a relative $\mathrm{M}$-complement of $\mathrm{N}$, then $\mathrm{N}$ is a relative $\mathrm{M}$-complement of $\mathrm{B}$, where a relative complement for $\mathrm{K}$ in $\mathrm{M}$ is any submodule $\mathrm{L}$ of $\mathrm{M}$ which is maximal with respect to the property $\mathrm{K} \cap \mathrm{L}=(0)[6]$.

\section{Proposition (1.4):}

Let $\mathrm{M}$ be an R-module, and let $(0) \neq \mathrm{C} \leq$ $\mathrm{M}$, then there exists an St-closed submodule $\overrightarrow{\mathrm{H}}$ in $\mathrm{M}$ such that $\mathrm{C} \leq_{\text {sem }} \mathrm{H}$.

\section{Proof:}

Consider the set $\mathrm{V}=\{\mathrm{K} \mid \mathrm{K}$ is a submodule of $M$ such that $C \leq$ sem $K\}$. It is clear that $\mathrm{V} \neq \varnothing$. By Zorn's Lemma, $\mathrm{V}$ has a maximal element say $\mathrm{H}$. In order to prove that $\mathrm{H}$ is an St-closed submodule in $\mathrm{M}$; assume that there exists a submodule $\mathrm{D}$ of $\mathrm{M}$ such that $\mathrm{H} \leq$ sem $\mathrm{D}$ $\leq \mathrm{M}$. Since $\mathrm{C} \leq_{\text {sem }} \mathrm{H}$ and $\mathrm{H} \leq_{\text {sem }} \mathrm{D}$, so by [11, Proposition (1.5)], $\mathrm{C} \leq$ sem D. But this a contradicts the maximality of $\mathrm{H}$, thus $\mathrm{H}=\mathrm{D}$. That is $\mathrm{H}$ is an St-closed submodule in $\mathrm{M}$ with $\mathrm{C} \leq_{\text {sem }} \mathrm{H}$.

We cannot prove the transitive property for St-closed submodules. However under some conditions we can prove this property as we see in the following result.

\section{Proposition (1.5):}

Let $\mathrm{A}$ and $\mathrm{B}$ be submodules of an R-module C. If $A$ is an St-closed in B and B is an St-closed in C, then A is St-closed in C provided that $\mathrm{B}$ contained in (or containing) any semi-essential extension of $\mathrm{A}$.

\section{Proof:}

Let $\mathrm{L} \leq \mathrm{C}$ such that $\mathrm{A} \leq$ sem $\mathrm{L} \leq \mathrm{C}$. By assumption we have two cases: If $\mathrm{L} \leq \mathrm{B}$, since $\mathrm{A}$ is an St-closed submodule in $\mathrm{B}$ then $\mathrm{A}=\mathrm{L}$, hence $\mathrm{A}$ is an St-closed submodule in C. If $\mathrm{B} \leq \mathrm{L}$, since $\mathrm{A} \leq_{\text {sem }} \mathrm{L}$, so by [2, Proposition 4], $\mathrm{B} \leq$ sem L. But B is an St-closed in C, thus 
$\mathrm{B}=\mathrm{L}$. That is $\mathrm{A} \leq$ sem $\mathrm{B}$. On the other hand, A is an St-closed submodule in $\mathrm{B}$, so $\mathrm{A}=\mathrm{B}$, hence $\mathrm{A}$ is an St-closed submodule in $\mathrm{C}$.

Recall that an R-module $\mathrm{M}$ is called chained if for each submodules $\mathrm{A}$ and $\mathrm{B}$ of $\mathrm{M}$ either $\mathrm{A} \leq \mathrm{B}$ or $\mathrm{B} \leq \mathrm{A}[13]$.

\section{Corollary (1.6):}

Let $\mathrm{M}$ be a chained module, and let $\mathrm{A}$ and $B$ be submodules of $M$ such that $A \leq B \leq M$. if A is an St-closed submodule in B and B is an St-closed submodule in $\mathrm{M}$ then $\mathrm{A}$ is an St-closed submodule in $\mathrm{M}$.

\section{Proof:}

Let $\mathrm{L} \leq \mathrm{M}$ such that $\mathrm{A} \leq$ sem $\mathrm{L} \leq \mathrm{M}$. since $\mathrm{M}$ is a chained module, then either $\mathrm{L} \leq \mathrm{B}$ or $\mathrm{B} \leq \mathrm{L}$, and the result follows as the same argument which used in the proof of the Proposition (1.5).

We can put other condition to get the transitive property of St-closed submodules, but before that we need to recall some definitions and give some remarks.

Recall that a nonzero R-module $\mathrm{M}$ is called fully essential, if every nonzero semi-essential submodule of $M$ is essential submodule of $M$ [12], and an R-module $M$ is called fully prime, if every proper submodule of $\mathrm{M}$ is a prime submodule [3], and every fully prime module is a fully essential module [11].

\section{Proposition (1.7):}

Let $\mathrm{N}$ be a nonzero closed submodule of an R-module $M$. If every semi-essential extensions of $\mathrm{N}$ is a fully essential submodule of $\mathrm{M}$, then $\mathrm{N}$ is an St-closed submodule in $\mathrm{M}$.

\section{Proof:}

Let $\mathrm{N}$ be a nonzero closed submodule of $\mathrm{M}$, and let $\mathrm{L} \leq \mathrm{M}$ such that $\mathrm{N} \leq$ sem $\mathrm{L} \leq \mathrm{M}$. By assumption $\mathrm{L}$ is a fully essential module, therefore $\mathrm{N} \leq_{e}$ L. But $\mathrm{N} \leq_{\text {co }} \mathrm{M}$, thus $\mathrm{N}=\mathrm{L}$. That is $\mathrm{N} \leq \mathrm{stc}$ M.

\section{Remark (1.8):}

If an R-module $\mathrm{M}$ is fully prime, then every nonzero closed submodule in $\mathrm{M}$ is an St-closed submodule in $\mathrm{M}$.

\section{Proof:}

Let $\mathrm{N}$ be a nonzero closed submodule of $\mathrm{M}$, and let $\mathrm{N} \leq$ sem $\mathrm{L} \leq \mathrm{M}$. Then by [11, Proposition (2.1)], $\mathrm{N} \leq_{\mathrm{e}} \mathrm{L}$. But $\mathrm{N} \leq_{\text {co }} \mathrm{M}$, thus $\mathrm{N}=\mathrm{L}$, and we are don.

\section{Proposition (1.9):}

Let $\mathrm{C}$ be an R-module and let $(0) \neq \mathrm{A} \leq$ $\mathrm{B} \leq \mathrm{C}$. Assume that every semi-essential extension of $\mathrm{A}$ is a fully essential submodule of $\mathrm{M}$. If $\mathrm{A} \leq_{\mathrm{Stc}} \mathrm{B}$ and $\mathrm{B} \leq_{\mathrm{Stc}} \mathrm{C}$, then $\mathrm{A} \leq_{\mathrm{Stc}} \mathrm{C}$.

\section{Proof:}

Since $\mathrm{A} \leq_{\mathrm{Stc}} \mathrm{B}$ and $\mathrm{B} \leq_{\mathrm{Stc}} \mathrm{C}$, then by Remark (1.3) (1), $\mathrm{A} \leq_{c} \mathrm{~B}$ and $\mathrm{B} \leq_{c} \mathrm{C}$. this implies that $\mathrm{A} \leq_{\mathrm{c}} \mathrm{C}$, [6, Proposition (1.5), P.18]. And by Proposition (1.7), A is an St-closed submodule in C.

In a similar proof of Proposition (1.9), and by using Remark (1.8) instead of Proposition (1.7) we can prove the following.

\section{Proposition (1.10):}

Let $\mathrm{M}$ be a fully prime module, and let (0) $\neq \mathrm{A} \leq_{\text {stc }} \mathrm{B}$ and $\mathrm{B} \leq_{\text {stc }} \mathrm{M}$, then $\mathrm{A} \leq_{\text {Stc }} \mathrm{M}$.

The following remarks verify the hereditary of St-closed property between two submodules of an R-module M.

\section{Remark (1.11):}

Let $\mathrm{A}$ and $\mathrm{B}$ are submodules of an $\mathrm{R}$-module $\mathrm{M}$ such that $\mathrm{A} \leq \mathrm{B} \leq \mathrm{M}$. If $\mathrm{B}$ is an St-closed submodule in $\mathrm{M}$, then A need not be St-closed submodule in M. For example; the $\mathrm{Z}$-module $\mathrm{Z}$ is an St-closed submodule of $\mathrm{Z}$ and $2 Z \leq Z$, while $2 Z$ is not St-closed submodule in $\mathrm{Z}$.

\section{Remark (1.12):}

If $\mathrm{A}$ and $\mathrm{B}$ are submodules of an R-module $M$ such that $A \leq B \leq M$. If $A$ is an St-closed submodule in $\mathrm{M}$, then $\mathrm{B}$ need not be St-closed submodule in $\mathrm{M}$. For example; the $\mathrm{Z}$-module $\mathrm{Z}$ and the submodules $\mathrm{A}=(0)$ and $\mathrm{B}=2 \mathrm{Z}$. Note that (0) is an St-closed submodule in $\mathrm{Z}$, but $2 \mathrm{Z}$ is not St-closed submodule in $\mathrm{Z}$, since $2 \mathrm{Z}$ is a semi-essential submodule of $\mathrm{Z}$.

\section{Proposition (1.13):}

If every submodule of $\mathrm{M}$ is an St-closed, then every submodule of $\mathrm{M}$ is a direct summand of $\mathrm{M}$.

\section{Proof:}

Since every submodule of $M$ is an St-closed, and by Remarks (1.3) (1), every St-closed submodule is a closed, so every submodule of $\mathrm{M}$ is a closed. Hence the result follows from [8, Exercises (6- c), P.139].

It is well known that the intersection of two closed submodules need not be closed 
submodule for example: Consider the $\mathrm{Z}$-module $\mathrm{M}=\mathrm{Z} \oplus \mathrm{Z}_{2}$, If we take $\mathrm{A}=\langle(1, \overline{0})$ $>$ and $\mathrm{B}=\langle(1, \overline{1})\rangle$, it is clear that both of them are direct summands of $M$, so they are closed in $\mathrm{M}$. But $\mathrm{A} \cap \mathrm{B}=\langle(2, \overline{0})\rangle$ and $(A \cap B) \leq_{e} B$, that is $A \cap B$ is not closed in $M$ [6, Example (1.6), P.19]. However, we have the following.

\section{Proposition (1.14):}

Let A and B be St-closed submodules in an R-module $\mathrm{M}$, then $\mathrm{A} \cap \mathrm{B}$ is an St-closed submodule in $\mathrm{M}$.

\section{Proof:}

Let $\mathrm{L} \leq \mathrm{M}$ such that $\mathrm{A} \cap \mathrm{B} \leq$ sem $\mathrm{L} \leq \mathrm{M}$. By [2, Corollary (6), P.49] A $\leq_{\text {sem } L}$ and B $\leq_{\text {sem }}$ L. Since $\mathrm{A}$ and $\mathrm{B}$ are St-closed submodules in $\mathrm{M}$, then $\mathrm{A}=\mathrm{L}=\mathrm{B}$, hence $\mathrm{A} \cap \mathrm{B}=\mathrm{L}$.

\section{Proposition (1.15):}

Let $\mathrm{M}$ be an $\mathrm{R}$-module, and let $\mathrm{A}$ and $\mathrm{B}$ be submodules of $\mathrm{M}$ such that $\mathrm{A} \leq \mathrm{B} \leq \mathrm{M}$. If $\mathrm{A}$ is an St-closed submodule in $\mathrm{M}$, then $\mathrm{A}$ is an St-closed submodule in B.

\section{Proof:}

Suppose that $\mathrm{A} \leq \leq_{\text {sem }} \mathrm{L} \leq \mathrm{B}$, so $\mathrm{L} \leq \mathrm{M}$. But $\mathrm{A}$ is an St-closed submodule in $\mathrm{M}$, therefore $\mathrm{A}=\mathrm{L}$.

\section{Corollary (1.16):}

Let $\mathrm{A}$ and $\mathrm{B}$ be submodules of an R-module M. If $A \cap B$ is an St-closed submodule in $M$, then $\mathrm{A} \cap \mathrm{B}$ is an St-closed submodule in $\mathrm{A}$ and $\mathrm{B}$.

\section{Corollary (1.17):}

If $\mathrm{N}$ and $\mathrm{K}$ are St-closed submodules in an R-module $\mathrm{M}$, then $\mathrm{N}$ and $\mathrm{K}$ are St-closed submodules in $\mathrm{N}+\mathrm{K}$.

\section{Proof:}

Since $\mathrm{N} \leq \mathrm{N}+\mathrm{K} \leq \mathrm{M}$, so by Proposition (1.15) we are done.

We can proof the following proposition by using [12, Lemma (1.15)]. In fact this Lemma in [12] is true when we instead the condition "fully prime" by the condition "fully essential".

\section{Proposition (1.18):}

Let $\mathrm{M}=\mathrm{M}_{1} \oplus \mathrm{M}_{2}$ be a fully essential R-module where $\mathrm{M}_{1}$ and $\mathrm{M}_{2}$ be submodules, and let $\mathrm{A}$ and $\mathrm{B}$ be nonzero submodules of $\mathrm{M}_{1}$ and $\mathrm{M}_{2}$ respectively. If $\mathrm{A}$ and $\mathrm{B}$ are St-closed submodules in $\mathrm{M}_{1}$ and $\mathrm{M}_{2}$ respectively. Then $\mathrm{A} \oplus \mathrm{B}$ is an St-closed submodule in $\mathrm{M}_{1} \oplus \mathrm{M}_{2}$, provided that ann $\mathrm{M}_{1}+$ ann $\mathrm{M}_{2}=\mathrm{R}$.

\section{Proof:}

Assume that $A \oplus B \leq$ sem $L \leq M$. Since ann $\mathrm{M}_{1}+$ ann $\mathrm{M}_{2}=\mathrm{R}$, so by the same proof of [1, Proposition (4.2)], $\mathrm{L}=\mathrm{L}_{1} \oplus \mathrm{L}_{2}$, where $\mathrm{L}_{1} \leq \mathrm{M}_{1}$ and $\mathrm{L}_{2} \leq \mathrm{M}_{2}$. Therefore $\mathrm{A} \oplus \mathrm{B} \leq_{\text {sem }} \mathrm{L}_{1}$ $\oplus \mathrm{L}_{2}$, and by [12, Lemma (1.15)], $\mathrm{A} \leq_{\mathrm{sem}} \mathrm{L}_{1}$ and $\mathrm{B} \leq_{\text {sem }} \mathrm{L}_{2}$. But both of $\mathrm{A}$ and $\mathrm{B}$ are St-closed submodules in $\mathrm{M}$. So that $\mathrm{A}=\mathrm{L}_{1}$ and $\mathrm{B}=\mathrm{L}_{2}$, hence $\mathrm{A} \oplus \mathrm{B}=\mathrm{L}_{1} \oplus \mathrm{L}_{2}$.

\section{Proposition (1.19):}

Let $\mathrm{M}=\mathrm{M}_{1} \oplus \mathrm{M}_{2}$ be an R-module where $\mathrm{M}_{1}$ and $\mathrm{M}_{2}$ be submodules of $\mathrm{M}$, and let $\mathrm{A}, \mathrm{B}$ be St-closed submodule in $\mathrm{M}_{1}$ and $\mathrm{M}_{2}$ respectively. Then $\mathrm{A} \oplus \mathrm{B}$ is an St-closed submodule in $\mathrm{M}_{1} \oplus \mathrm{M}_{2}$, provided that aan $\mathrm{M}_{1}+$ ann $\mathrm{M}_{2}=\mathrm{R}$. And all semi essential extensions of $\mathrm{A} \oplus \mathrm{B}$ are fully essential modules.

\section{Proof:}

Assume that $\mathrm{A} \oplus \mathrm{B} \leq$ sem $\mathrm{L} \leq \mathrm{M}$. By the same argument of Proposition (1.18) we have $\mathrm{A} \oplus \mathrm{B} \leq_{\mathrm{sem}} \mathrm{L}_{1} \oplus \mathrm{L}_{2}$, where $\mathrm{L}=\mathrm{L}_{1} \oplus \mathrm{L}_{2}$. Since $\mathrm{L}$ is a fully essential module, then $\mathrm{A} \oplus \mathrm{B} \leq_{\mathrm{e}} \mathrm{L}_{1} \oplus \mathrm{L}_{2}$, this implies that $\mathrm{A} \leq_{\mathrm{e}} \mathrm{L}_{1}$ and $\mathrm{B} \leq_{\mathrm{e}} \mathrm{L}_{2}$. It is clear that both of $\mathrm{A}$ and $\mathrm{B}$ are closed submodules in $\mathrm{M}$, thus $\mathrm{A}=\mathrm{L}_{1}$ and $\mathrm{B}=\mathrm{L}_{2}$, hence $\mathrm{A} \oplus \mathrm{B}=\mathrm{L}_{1} \oplus \mathrm{L}_{2}$.

\section{Theorem (1.20):}

Let $\mathrm{M}=\mathrm{M}_{1} \oplus \mathrm{M}_{2}$ be a fully prime R-module where $\mathrm{M}_{1}$ and $\mathrm{M}_{2}$ be submodules of $\mathrm{M}$ and let $\mathrm{A}, \mathrm{B}$ be nonzero submodules of $\mathrm{M}_{1}$ and $\mathrm{M}_{2}$ respectively. Then $\mathrm{A} \oplus \mathrm{B}$ is an St-closed submodule in $\mathbf{M}_{1} \oplus \mathrm{M}_{2}$ if and only if $\mathrm{A}$ and $\mathrm{B}$ are St-closed submodules in $\mathrm{M}_{1}$ and $\mathrm{M}_{2}$ respectively.

\section{Proof:}

$\Rightarrow$ ) Assume that $A \leq$ sem $K \leq M_{1}$. Since $\mathrm{B} \leq_{\text {sem }} \mathrm{B}$, we can easily show that $\mathrm{K} \oplus \mathrm{B}$ is a fully prime module. In fact if $\mathrm{X}$ is a proper submodule of $K \oplus B$, and since $M$ is a fully prime module, then $\mathrm{X}$ is a prime submodule of M. By [7, Lemma (3.7)], $\mathrm{X}$ is a prime submodule of $\mathrm{K} \oplus \mathrm{B}$, and by [12, Lemma (1.15)], $\mathrm{A} \oplus \mathrm{B} \leq_{\text {sem }} \mathrm{K} \oplus \mathrm{B} \leq \mathrm{M}$. But $\mathrm{A} \oplus \mathrm{B} \leq_{\text {Stc }} \mathrm{M}$, thus $\mathrm{A} \oplus \mathrm{B}=\mathrm{K} \oplus \mathrm{B}$, that is 
$\mathrm{A}=\mathrm{K}$. In similar way we can prove that $\mathrm{B} \leq$ Stc $\mathrm{M}$.

$\Leftarrow)$ Since in a fully prime module the St-closed submodule and closed submodule are equivalent, so the result follows from [6, Exercises (15), P.20].

Recall that the prime radical of an $\mathrm{R}$-module $\mathrm{M}$ is denoted by $\operatorname{rad}(\mathrm{M})$, and it is the intersection of all prime submodules of $\mathrm{M}$ [10].

\section{Proposition (1.21):}

Let $\mathrm{f}: \mathrm{M} \rightarrow \mathrm{M}^{\prime}$ be an R-epimorphism from an R-module $\mathrm{M}$ to an R-module $\mathrm{M}^{\prime}$, and let $\mathrm{B}$ be a submodule of $M$ such that ker $f \subseteq \operatorname{rad}(\mathrm{M})$ $\cap B$. If $B$ is an St-closed submodule in $M$ then $\mathrm{f}(\mathrm{B})$ is an St-closed submodule in $\mathrm{M}^{\prime}$.

\section{Proof:}

Let $\mathrm{K}^{\prime}$ be a submodule of $\mathrm{M}^{\prime}$ such that $\mathrm{f}(\mathrm{B})$ $\leq$ sem $K^{\prime} \leq M^{\prime}$. Since ker $f \subseteq \operatorname{rad}(M)$, then $\mathrm{f}^{-1} \mathrm{f}(\mathrm{B}) \leq$ sem $\mathrm{f}^{-1}\left(\mathrm{~K}^{\prime}\right) \leq \mathrm{M}$ [2]. We can easily show that $\mathrm{f}^{-1} \mathrm{f}(\mathrm{B})=\mathrm{B}$ since ker $\mathrm{f} \subseteq \mathrm{B}$. This implies that $\mathrm{B} \leq \leq_{\operatorname{sem}} \mathrm{f}^{-1}\left(\mathrm{~K}^{\prime}\right)$. But $\mathrm{B}$ is an St-closed submodule in $M$, then $B=f^{-1}\left(K^{\prime}\right)$. Since $f$ is epimorphism so $f(B)=K^{\prime}$, and we are done.

\section{Corollary (1.22):}

Let $\mathrm{A}$ and $\mathrm{B}$ be submodules of an R-module $\mathrm{M}$, such that $\mathrm{A} \subseteq \operatorname{rad}(\mathrm{M}) \cap \mathrm{B}$. if $\mathrm{B}$ is an St-closed submodule in $M$, then $\frac{B}{A}$ is an St-closed submodule in $\frac{\mathrm{M}}{\mathrm{A}}$.

Recall that a singular submodule defined by $\mathrm{Z}(\mathrm{M})=\left\{\mathrm{x} \in \mathrm{M}: \operatorname{ann}(\mathrm{x}) \leq_{\mathrm{e}} \mathrm{R}\right\}$. If $\mathrm{Z}(\mathrm{M})=\mathrm{M}$, then $\mathrm{M}$ is called the singular module. If $\mathrm{Z}(\mathrm{M})$ $=0$ then $\mathrm{M}$ is called a nonsingular module, [6]. A submodule $\mathrm{N}$ of an $\mathrm{R}$-module $\mathrm{M}$ is called y-closed submodule of $M$, if $\frac{M}{N}$ is a nonsingular module [6, P.42]. We cannot find a direct relation between St-closed and $\mathrm{y}$-closed submodules. However, under some conditions we can find some cases of this relationship as the following proposition shows.

\section{Proposition (1.23):}

If $\mathrm{M}$ is a fully prime $\mathrm{R}$-module, then every nonzero y-closed submodule is an St-closed submodule.

\section{Proof:}

Let $\mathrm{A}$ be a nonzero y-closed submodule in $\mathrm{M}$, then by [9, Remarks and Examples (2.1.1)
(3)], $\mathrm{A}$ is a closed submodule in $\mathrm{M}$ and by Remark (1.8), A is an St-closed submodule in M.

\section{Proposition (1.24):}

Let $\mathrm{M}$ be a nonsingular $\mathrm{R}$-module, if a submodule $\mathrm{N}$ of $\mathrm{M}$ is an St-closed, then $\mathrm{N}$ is a $\mathrm{y}$-closed submodule.

\section{Proof:}

Let $\mathrm{N}$ be an St-closed submodule in $\mathrm{M}$, by Remarks (1.3) (1) $\mathrm{N}$ is a closed submodule in $\mathrm{M}$. But $\mathrm{M}$ is a nonsingular module, so by [9, Proposition (2.1.2)], $\mathrm{N}$ is a y-closed submodule of $\mathrm{M}$.

\section{Another proof:}

Assume that $\mathrm{M}$ is a nonsingular R-module, and let $\mathrm{N}$ be an St-closed submodule in $\mathrm{M}$. Let $\mathrm{Z}\left(\frac{\mathrm{M}}{\mathrm{N}}\right) \equiv \frac{\mathrm{B}}{\mathrm{N}}$, where $\mathrm{B}$ is a submodule of $\mathrm{M}$ with $\mathrm{N} \leq \mathrm{B}$. Clearly $\frac{\mathrm{B}}{\mathrm{N}}$ is a singular module. Now $\mathrm{N}$ $\leq \mathrm{B}$ and $\mathrm{M}$ is a nonsingular module, therefore $\mathrm{B}$ is a nonsingular submodule of $\mathrm{M}$. Then by [6, Proposition (1.21), P.32], $\mathrm{N} \leq \mathrm{e}$ B, hence $\mathrm{N}$ $\leq$ sem B. But A is an St-closed submodule in M, thus $N=B$, and $Z\left(\frac{M}{N}\right)=(0)$. So $\frac{M}{N}$ is a nonsingular module, and by the definition of y-closed submodule, $\mathrm{N}$ is a $\mathrm{y}$-closed submodule in $\mathrm{M}$.

\section{Theorem (1.25):}

Let $\mathrm{M}$ be a fully prime R-module, and let $\mathrm{N}$ be a nonzero submodule of $\mathrm{M}$. Consider the following statement:

1. $\mathrm{N}$ is a y-closed submodule.

2. $\mathrm{N}$ is a closed submodule.

3. $\mathrm{N}$ is an St-closed submodule.

Then (1) $\Rightarrow(2) \Leftrightarrow$ (3), and if $M$ is a nonsingular module, then (3) $\Rightarrow$ (1)

\section{Proof:}

(1) $\Rightarrow$ (2) [9, Remarks and Examples (2.1.1), 3]

(2) $\Leftrightarrow$ (3) Since $M$ is a fully prime module then by, Remark (1.8), $\mathrm{N}$ is an St-closed submodule. The converse is clear.

(3) $\Rightarrow$ (1) Since $M$ is a nonsingular module, then by Proposition (1.24), $\mathrm{N}$ is a y-closed submodule.

\section{$\mathrm{S}_{2}$ : St-closed submodules in multiplication modules}

In this section we study the behavior of the St-closed submodules in the class of 
multiplication modules. Also we study the hereditary property of the St-closed submodules between R-modules and R itself.

Recall that An R-module $\mathrm{M}$ is called multiplication module, if every submodule $\mathrm{N}$ of $\mathrm{M}$ is of the form IM for some ideal I of $\mathrm{R}$ [4]. Recall that a nonzero prime submodule $\mathrm{N}$ of an R-module $\mathrm{M}$ is called minimal prime submodule of $\mathrm{M}$ if whenever $\mathrm{P}$ is a nonzero prime submodule of $\mathrm{M}$ such that $\mathrm{P} \subseteq \mathrm{N}$, then $\mathrm{P}=\mathrm{N}[5]$.

\section{Proposition (2.1):}

Let $M$ be a faithful and multiplication $\mathrm{R}$-module, and let $\mathrm{N}$ be a nonzero prime submodule of $\mathrm{M}$. If $\mathrm{N}$ is an St-closed submodule in $\mathrm{M}$, then $\mathrm{N}$ is a minimal prime submodule of $\mathrm{M}$.

\section{Proof:}

Suppose that $\mathrm{N}$ is not minimal prime submodule of M. By [2, Prop(3), P.53], $\mathrm{N}$ is a semi-essential submodule of $\mathrm{M}$. But $\mathrm{N}$ is an St-closed, thus $\mathrm{N}=\mathrm{M}$. On the other hand $\mathrm{N}$ is a prime submodule that is $\mathrm{N}$ must be a proper submodule of $\mathrm{M}$, so we get a contradiction.

\section{Proposition (2.2):}

Let $\mathrm{M}$ be a nonzero multiplication R-module with only one nonzero maximal submodule $\mathrm{N}$, then $\mathrm{N}$ cannot be St-closed submodule in $\mathrm{M}$.

\section{Proof:}

Assume that $\mathrm{N}$ is an St-closed submodule in M, so by [11, Proposition (2.13)] $\mathrm{N} \leq$ sem M. By Examples and Remarks (1.2) (4) $\mathrm{N}=\mathrm{M}$, but this contradicts with a maximality of $\mathrm{N}$, therefore $\mathrm{N}$ is not St-closed submodule in $\mathrm{M}$.

\section{Remark (2.3):}

In Proposition (2.2), we get the same result when we replace the condition "nonzero multiplication" by the condition "finitely generated", and by using [11, Proposition (2.14)] instead of [11, Proposition (2.13)].

\section{Proposition (2.4):}

Let $M$ be a faithful and multiplication module such that $\mathrm{M}$ satisfies the condition (*), if $\mathrm{I}$ is an St-closed ideal in $\mathrm{J}$ then IM is an St-closed submodule in JM.

Condition (*): For any R-module $\mathrm{M}$ and any ideals $\mathrm{P}$ and $\mathrm{K}$ of $\mathrm{R}$ such that $\mathrm{P}$ is a prime ideal of $\mathrm{K}$, implies that $\mathrm{PM}$ is a prime submodule of KM.

\section{Proof:}

Assume that IM $\leq$ sem $\mathrm{L} \leq \mathrm{JM}$. We have to show that $\mathrm{IM}=\mathrm{L}$. Since $\mathrm{M}$ is a multiplication module, then $\mathrm{L}=\mathrm{TM}$ for some ideal $\mathrm{T}$ of $\mathrm{R}$. Now IM $\leq_{\text {sem }} \mathrm{TM} \leq \mathrm{JM}$, since $\mathrm{M}$ is a faithful and multiplication module and satisfying the condition (*), so by [11, Proposition (2.10)] I $\leq_{\text {sem }} \mathrm{T} \leq \mathrm{J}$. But $\mathrm{I}$ is an St-closed ideal in J, then $\mathrm{I}=\mathrm{T}$. This implies that $\mathrm{IM}=\mathrm{TM}=\mathrm{L}$, hence IM is an St-closed submodule in JM.

\section{Proposition (2.5):}

Let $\mathrm{M}$ be a finitely generated, faithful and multiplication module. If IM is an St-closed submodule in JM, then I is an St-closed ideal in $\mathrm{J}$.

\section{Proof:}

Assume that $\mathrm{I} \leq_{\text {sem }} \mathrm{E} \leq \mathrm{J}$, then by $[11$, Proposition (2.11)] IM $\leq$ sem EM $\leq \mathrm{JM}$. Since IM is St-closed in JM, then IM = EM. This implies that $\mathrm{I}=\mathrm{E}$, [5, Theorem (3.1)]. Thus $\mathrm{I}$ is an St-closed submodule in $\mathrm{J}$.

From Proposition (2.4) and Proposition (2.5) we get the following theorem.

Theorem (2.6): Let $\mathrm{M}$ be a finitely generated, faithful and multiplication module such that $\mathrm{M}$ satisfies the condition $(*)$, then I is an Stclosed ideal in $\mathbf{J}$ if and only if IM is an Stclosed submodule in JM.

\section{Corollary (2.7):}

Let $M$ be a finitely generated, faithful and multiplication $\mathrm{R}$-module, and let $\mathrm{N}$ be a submodule of $\mathrm{M}$. If $\mathrm{M}$ satisfies the condition $(*)$, then the following statements are equivalent:

1. $\mathrm{N}$ is an St-closed submodule in $\mathrm{M}$.

2. $\left(N_{R} M\right)$ is an St-closed ideal in $R$.

3. $\mathrm{N}=\mathrm{IM}$ for some St-closed ideal I in R.

\section{Proof:}

(1) $\Rightarrow$ (2) Assume that $\mathrm{N}$ is an St-closed submodule in $\mathrm{M}$. Since $\mathrm{M}$ is a multiplication module, then $\mathrm{N}=\left(\mathrm{N}_{\mathrm{R}}^{\vdots} \mathrm{M}\right) \mathrm{M}$ [5]. Put $\left(\mathrm{N}_{\mathrm{R}}^{\vdots} \mathrm{M}\right) \equiv$ $\mathrm{I}$, so we get IM is an St-closed submodule in M. By Theorem (2.6), I is an St-closed ideal in $\mathrm{R}$.

(2) $\Rightarrow$ (3) Since $M$ is a multiplication module, then $\mathrm{N}=\left(\mathrm{N}_{\mathrm{R}} \mathrm{M}\right) \mathrm{M}$ [5], and we are done.

(3) $\Rightarrow$ (1) Since I is an St-closed ideal in R, so by Theorem (2.6), IM $=\mathrm{N}$ is an St-closed submodule in $\mathrm{RM}=\mathrm{M}$. 


\section{S3:Chain condition on St-closed submodules}

In this section we study the chain condition on St-closed submodules, we give some results and examples about this concept. We start by the following definitions.

\section{Definition (3.1):}

An R-module $\mathrm{M}$ is said to have the ascending chain condition of St-closed submodules (briefly ACC on St-closed submodules), if every ascending chain $\mathrm{A}_{1} \subseteq \mathrm{A}_{2} \subseteq \ldots$ of St-closed submodules in $\mathrm{M}$ is finite. That is there exists $\mathrm{k} \in \mathrm{Z}_{+}$such that $\mathrm{A}_{\mathrm{n}}=\mathrm{A}_{\mathrm{k}}$ for all $\mathrm{n} \geq \mathrm{k}$.

\section{Definition (3.2):}

An R-module $\mathrm{M}$ is said to have the descending chain condition of St-closed submodules (briefly DCC on St-closed submodules), if every descending chain $\mathrm{A}_{1} \supseteq$ $\mathrm{A}_{2} \supseteq \ldots$ of St-closed submodules in $\mathrm{M}$ is finite. That is there exists $\mathrm{k} \in \mathrm{Z}_{+}$such that $A_{n}=A_{k}$, for all $n \geq k$.

\section{Examples and Remarks (3.3):}

1) Every Noetherian (respectively Artinian) module satisfies ACC (DCC) on St-closed submodules.

2) Every uniform modules satisfies $A C C$ on St-closed submodules. In fact in a uniform module, the only St-closed submodules are only $\mathrm{M}$ and sometime (0).

3) If $M$ satisfies ACC on closed submodules, then $M$ satisfies ACC on St-closed submodules.

\section{Proof:}

let $A_{1} \subseteq A_{2} \subseteq \ldots$ be an ascending chain of St-closed submodules of M. Since every St-closed submodule is closed submodule, then $A_{i}$ is a closed submodule $\forall i=1,2, \ldots$. By assumption $M$ is satisfies ACC on closed submodule, so that $\exists \mathrm{k} \in \mathrm{Z}_{+}$such that $\mathrm{A}_{\mathrm{n}}=\mathrm{A}_{\mathrm{k}}$ $\forall \mathrm{n} \geq \mathrm{k}$. That is $\mathrm{M}$ satisfies ACC on St-closed submodules.

\section{Proposition (3.4):}

Let $\mathrm{M}$ be a finitely generated, faithful and multiplication R-module. Assume that $\mathrm{M}$ satisfies the condition (*), then $\mathrm{M}$ satisfies ACC on St-closed submodules, if and only if $\mathrm{R}$ satisfies ACC on St-closed ideals.
Proof:

$\Rightarrow$ ): Let $\mathrm{J}_{1} \subseteq \mathrm{J}_{2} \subseteq \ldots$ be an ascending chain of St-closed ideals in R. Since $\mathrm{J}_{\mathrm{i}}$ is an St-closed ideal in $\mathrm{R}$, then by Theorem (2.6), $\mathrm{J}_{\mathrm{i}} \mathrm{M}$ is an St-closed submodule in $\mathrm{M} \forall \mathrm{i}=1,2, \ldots$. Note that $\mathrm{J}_{1} \mathrm{M} \subseteq \mathrm{J}_{2} \mathrm{M} \subseteq \ldots$ be an ascending chain of St-closed submodules in M. But M satisfies ACC on St-closed submodules, so $\exists \mathrm{k} \in \mathrm{Z}_{+}$ such that $\mathrm{J}_{\mathrm{k}} \mathrm{M}=\mathrm{J}_{\mathrm{n}} \mathrm{M} \forall \mathrm{n} \geq \mathrm{k}$. But $\mathrm{M}$ is a finitely generated, faithful and multiplication module, then $\mathrm{J}_{\mathrm{k}}=\mathrm{J}_{\mathrm{n}} \forall \mathrm{n} \geq \mathrm{k}$ [5, Theorem (3.1)]. Therefore R satisfies ACC on St-closed ideals.

$\Leftarrow)$ : Let $\mathrm{A}_{1} \subseteq \mathrm{A}_{2} \subseteq \ldots$ be an ascending chain of St-closed submodules in M. Since $M$ is a multiplication module, then $\mathrm{A}_{\mathrm{i}}=\mathrm{J}_{\mathrm{i}} \mathrm{M}$ for some ideal $\mathrm{J}_{\mathrm{i}}$ of $\mathrm{R} \forall \mathrm{i}=1,2, \ldots$. It is clear that $\mathrm{J}_{1} \mathrm{M}$ $\subseteq \mathrm{J}_{2} \mathrm{M} \subseteq \ldots$, since $\mathrm{A}_{\mathrm{i}}$ is an St-closed submodule in $\mathrm{M} \forall \mathrm{i}=1,2, \ldots$ and $\mathrm{M}$ is a finitely generated, faithful and multiplication module and satisfying the condition $(*)$, so by Theorem (2.6), $\mathrm{J}_{\mathrm{i}}$ is an St-closed ideal in $\mathrm{R} \forall \mathrm{i}$ $1,2, \ldots$. By $\left[5\right.$, Theorem (3.1)], $\mathrm{J}_{1} \subseteq \mathrm{J}_{2} \subseteq \ldots$, but R satisfies ACC on St-closed ideals, therefore there exists $\mathrm{k} \in \mathrm{Z}_{+}$such that $\mathrm{J}_{\mathrm{n}}=\mathrm{J}_{\mathrm{k}} \forall$ $\mathrm{n} \geq \mathrm{k}$, so that $\mathrm{J}_{\mathrm{n}} \mathrm{M}=\mathrm{J}_{\mathrm{k}} \mathrm{M}$, for each $\mathrm{n} \geq \mathrm{k}$, thus $\mathrm{A}_{\mathrm{n}}=\mathrm{A}_{\mathrm{k}} \forall \mathrm{n} \geq \mathrm{k}$. That is $\mathrm{M}$ satisfies ACC on St-closed submodules.

\section{Proposition (3.5):}

Let $\mathrm{M}$ be a chained R-module, and let $\mathrm{A}$ be an St-closed submodule of $\mathrm{M}$. If $\mathrm{M}$ satisfies ACC on St-closed submodules, then A satisfies ACC on St-closed submodules.

\section{Proof:}

Assume that M satisfies ACC on St-closed submodules and $\mathrm{A}_{1} \subseteq \mathrm{A}_{2} \subseteq \ldots$, be ascending chain of St-closed submodules of A. Since A is an St-closed submodule of $\mathrm{M}$, and $\mathrm{M}$ is a chained module, so by Corollary (1.6), $\mathrm{A}_{\mathrm{i}}$ is an St-closed submodule of $M$. Hence $A_{1} \subseteq A_{2} \subseteq$ ..., be ascending chain of St-closed submodules of $\mathrm{M}$. By assumption there exists $\mathrm{k} \in \mathrm{Z}_{+}$such that $\mathrm{A}_{\mathrm{n}}=A_{\mathrm{k}} \forall \mathrm{n} \geq \mathrm{k}$, and we are done.

\section{Proposition (3.6):}

Let $\mathrm{M}$ be an R-module, and let $\mathrm{N}$ be a submodule of $\mathrm{M}$ such that $\mathrm{N} \subseteq \operatorname{rad}(\mathrm{M}) \cap \mathrm{H}$, where $H$ is any St-closed submodule in M. If $\frac{M}{N}$ satisfies ACC on St-closed submodules, then $\mathrm{M}$ is satisfies ACC on St-closed submodules. 


\section{Proof:}

Let $\mathrm{A}_{1} \subseteq \mathrm{A}_{2} \subseteq \ldots$ be an ascending chain of St-closed submodules in $M$. Since $A_{i}$ is an St-closed submodule in $\mathrm{M}$, and by assumption $\mathrm{N} \subseteq \operatorname{rad}(\mathrm{M}) \cap \mathrm{A}_{\mathrm{i}}$, for each $\mathrm{i} ; \mathrm{i}=1,2, \ldots$ so by Corollary (1.22), we get $\frac{A_{i}}{N}$ is an St-closed submodule in $\frac{\mathrm{M}}{\mathrm{N}}$ for each $\mathrm{i} ; \mathrm{i}=1,2, \ldots$. Hence $\frac{A_{1}}{N} \subseteq \frac{A_{2}}{N} \subseteq \ldots$ be ascending chain of St-closed submodules in $\frac{M}{N}$. Since $\frac{M}{N}$ is satisfied ACC on St-closed submodules, so there exists $\mathrm{k} \in \mathrm{Z}_{+}$ such that $\frac{A_{n}}{N}=\frac{A_{k}}{N} \forall n \geq k$. So that $A_{n}=A_{k}$ and we get the result.

\section{Proposition (3.7):}

Let $\mathrm{M}=\mathrm{M}_{1} \oplus \mathrm{M}_{2}$ be a fully essential R-module, where $\mathrm{M}_{1}$ and $\mathrm{M}_{2}$ are submodules. If $\mathrm{M}$ satisfies ACC on St-closed submodules, then $\mathrm{M}_{1}$ (or $\mathrm{M}_{2}$ ) satisfies ACC on nonzero St-closed submodules, provided that ann $\mathrm{M}_{1}+$ ann $\mathrm{M}_{2}=\mathrm{R}$.

\section{Proof:}

Let $\mathrm{A}_{1} \subseteq \mathrm{A}_{2} \subseteq \ldots$, be ascending chain of nonzero St-closed submodules of $\mathrm{M}_{1}$. If $\mathrm{M}_{2}$ is equal to zero then $\mathrm{M}=\mathrm{M}_{1}$, and this implies that $\mathrm{M}_{1}$ satisfies $\mathrm{ACC}$ on nonzero St-closed submodule. Otherwise, since $A_{i}$ is a nonzero St-closed submodule in $\mathrm{M}_{1}$, and $\mathrm{M}_{2}$ is an St-closed submodule in $\mathrm{M}_{2}$, So by Proposition (1.18), $A_{i} \oplus M_{2}$ is an St-closed submodule in $\mathrm{M} \forall \mathrm{i}=1,2, \ldots$. Since $M$ satisfies $\mathrm{ACC}$ on St-closed submodules, then there exists $\mathrm{k} \in \mathrm{Z}_{+}$ such that $A_{n} \oplus M_{2}=A_{k} \oplus M_{2} \forall n \geq k$. Thus $A_{n}=A_{k}, \forall n \geq k$. Similarity for $M_{2}$.

The converse of Proposition (3.7) is true when every closed submodule of $M$ is fully invariant as the following proposition shows.

\section{Proposition (3.8):}

Let $\mathrm{M}=\mathrm{M}_{1} \oplus \mathrm{M}_{2}$ be an R-module, where $\mathrm{M}_{1}$ and $\mathrm{M}_{2}$ are St-closed submodules in $\mathrm{M}$. If $\mathrm{M}_{\mathrm{i}}$ satisfies ACC on nonzero St-closed submodules, for each $\mathrm{i} ; \mathrm{i}=1,2$. Then $\mathrm{M}$ satisfies ACC on nonzero St-closed submodules, provided that every St-closed submodule of $\mathrm{M}$ is a fully invariant.

\section{Proof:}

Assume that $\mathrm{A}_{1} \subseteq \mathrm{A}_{2} \subseteq \ldots$ is an ascending chain of nonzero St-closed submodules in $\mathrm{M}$, and let $\pi_{\mathrm{i}}: \mathrm{M} \rightarrow \mathrm{M}_{\mathrm{i}}$ be the projection maps for each $\mathrm{j} \in \mathrm{J}$ where $\mathrm{J}=1,2, \ldots$. We claim that
$A_{j}=\left(A_{j} \cap M_{1}\right) \oplus\left(A_{j} \cap M_{2}\right)$. To verify that, let $\mathrm{x} \in \mathrm{A}_{\mathrm{j}}$ then $\mathrm{x}=\mathrm{m}_{1} \oplus \mathrm{m}_{2}$, where $\mathrm{m}_{1} \in \mathrm{M}_{1}$ and $\mathrm{m}_{2} \in \mathrm{M}_{2}$. Since $\mathrm{A}_{\mathrm{j}}$ is an St-closed submodule of $M$ for each $j \in J$, and by our assumption, $A_{j}$ is a fully invariant which implies that $\pi_{1}(\mathrm{x})=\mathrm{m}_{1} \in \mathrm{A}_{\mathrm{j}} \cap \mathrm{M}_{1}$ and $\pi_{2}(\mathrm{x})=\mathrm{m}_{2} \in \mathrm{A}_{\mathrm{j}} \cap$ $M_{2}$. So $x \in\left(A_{j} \cap M_{1}\right) \oplus\left(A_{j} \cap M_{2}\right)$. Thus $A_{j} \subseteq$ $\left(A_{j} \cap M_{1}\right) \oplus\left(A_{j} \cap M_{2}\right)$. But $\left(A_{j} \cap M_{1}\right) \oplus\left(A_{j}\right.$ $\left.\cap M_{2}\right) \subseteq A_{j}$, therefore $A_{j}=\left(A_{j} \cap M_{1}\right) \oplus\left(A_{j} \cap\right.$ $M_{2}$ ). Note that $A_{j}$ and $M_{i}$ are St-closed submodule in $\mathrm{M}$, so by Proposition (1.14), $\mathrm{A}_{\mathrm{j}}$ $\cap \mathrm{M}_{\mathrm{i}}$ is an St-closed submodule in M. Since $\mathrm{A}_{\mathrm{j}}$ $\cap \mathrm{M}_{\mathrm{i}} \leq \mathrm{M}_{\mathrm{i}} \leq \mathrm{M}$, then by Proposition (1.15), $\mathrm{A}_{\mathrm{j}}$ $\cap M_{i}$ is an St-closed submodules in $M_{i}$ for each $i=1,2$ and $j=1,2, \ldots$. We can easily show that $\left(A_{j} \cap M_{i}\right) \neq(0)$ for each $j=1,2, \ldots$ and $i=1,2$. In fact if $A_{j} \cap M_{i}=(0)$ for each $i=1,2$ and $j=1,2, \ldots$, then by using $A_{j}=\left(A_{j}\right.$ $\left.\cap M_{1}\right) \oplus\left(A_{j} \cap M_{2}\right)$, we get $A_{j}=(0)$, which is contradicts with our assumption. That is $\mathrm{A}_{\mathrm{j}} \cap \mathrm{M}_{\mathrm{i}}$ are nonzero St-closed submodules in $\mathrm{M}$ for all $\mathrm{i}, \mathrm{j}$. We have the following ascending chain of St-closed submodules in $\mathrm{M}_{\mathrm{i}},\left(\mathrm{A}_{1} \cap\right.$ $\left.\mathrm{M}_{\mathrm{i}}\right) \subseteq\left(\mathrm{A}_{2} \cap \mathrm{M}_{\mathrm{i}}\right) \subseteq \ldots, \forall \mathrm{i}=1,2$. But $\mathrm{M}_{\mathrm{i}}$ satisfies ACC on nonzero St-closed submodules, then for each $\mathrm{i}=1,2$, there exists $\mathrm{k}_{\mathrm{i}} \in \mathrm{Z}_{+}$such that $\mathrm{A}_{\mathrm{n}} \cap \mathrm{M}_{\mathrm{i}}=\mathrm{A}_{\mathrm{ki}} \cap \mathrm{M}_{\mathrm{i}} \forall \mathrm{n} \geq \mathrm{k}_{\mathrm{i}}$. Let $\mathrm{k}=\max \left\{\mathrm{k}_{1}, \mathrm{k}_{2}\right\}$. So $\mathrm{A}_{\mathrm{n}}=\left(\mathrm{A}_{\mathrm{n}} \cap \mathrm{M}_{1}\right) \oplus$ $\left(A_{n} \cap M_{2}\right)=\left(A_{k} \cap M_{1}\right) \oplus\left(A_{k} \cap M_{2}\right)=A_{k}$ for each $n \geq k$. Thus $M$ satisfies ACC on nonzero St-closed submodules.

\section{Remark (3.9):}

We can generalize Proposition (3.8) for finite index $I$ of the direct sum of R-modules.

\section{Reference}

[1] Abbas M.S., "on fully stable modules", Ph.D. Thesis, University of Baghdad, Iraq, 1990.

[2] Ali. S. Mijbass, and Nada. K. Abdullah, "Semi-essential submodule and semiuniform modules", J. of Kirkuk University-Scientific studies, 4 (1), 2009.

[3] Behboodi M Karamzadeh O. A. S, and Koohy H., "Modules whose certain submodule are prime", Vietnam J. of Mathematics, 32(3): 303-317, 2004.

[4] Barnard A., "Multiplication modules", J. Algebra, 71: 174-178, 1981. 
[5] El-Bast Z. A. and Smith P. F., "Multiplication modules", Comm. In Algebra, 16: 755-779, 1988.

[6] Goodearl K. R., "Ring theory", Marcel Dekker, New York, 1976.

[7] Ibrahiem T. A., "Prime extending module and S-prime module", J. of Al-Nahrain Univ., Vol. 14(4), 166-170, 2011.

[8] Kasch F., "Modules and rings", London: Academic Press, 1982.

[9] Lamyaa Hussein Sahib, "Extending, injectivity and chain condition on y-closed submodules", Thesis, University of Baghdad, Iraq, 2012.

[10] Larsen, M. D. and McCarthy, P. J., Multiplicative theory of ideals, Acad. press, New York and London, 1971.

[11] Muna A. Ahmed, and Maysaa, R. Abbas, On semi-essential submodules, Ibn Al-Haitham J. for Pure \& Applied Science, Vol. 28 (1), 179- 185, 2015.

[12] Muna A. Ahmed, and Shireen O. Dakheen, S-maximal submodules, J. of Baghdad for Science, Vol. 12 (1), 210220, 2015.

[13] Osofsky, B.L. "A construction of nonstandard uniserial modules over valuation domain. Bulletin Amer. Math. Soc $25: 89-97,1991$.

[14] Saymeh S. A., on prime R-submodules, Univ. Ndc. Tucuma'n Rev. Ser. A29, 129136, 1979.

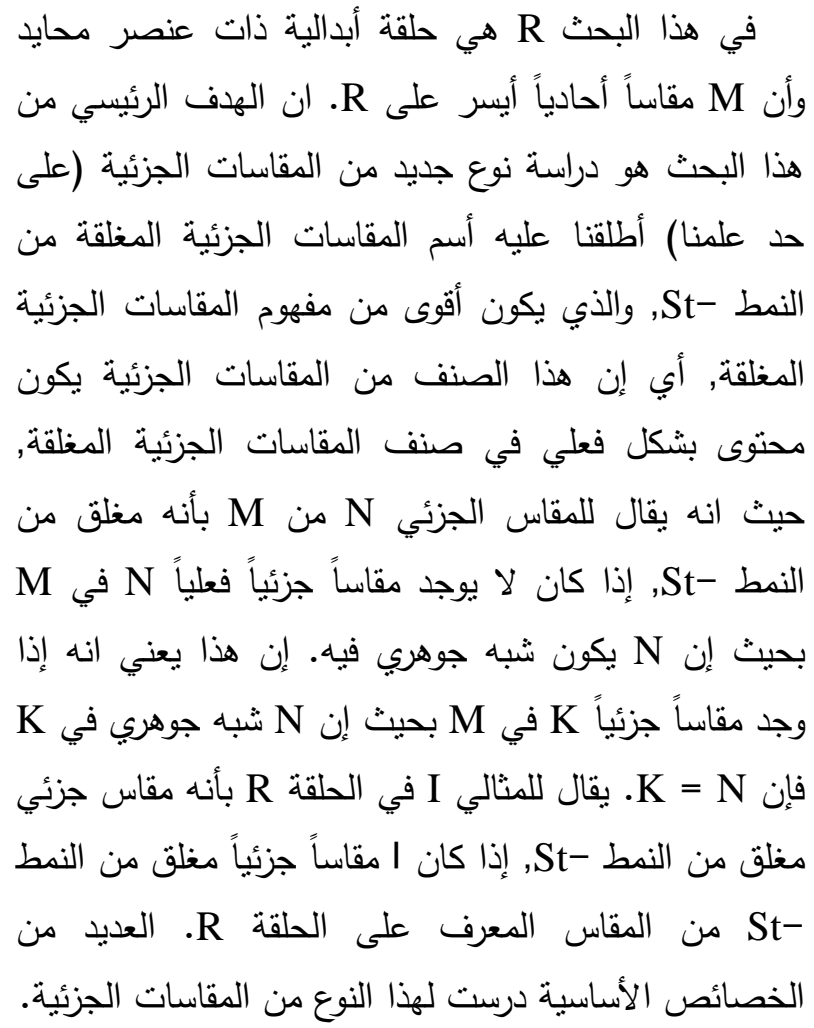

